\title{
Prioritizing Equity in a Time of Scarcity: The COVID-19 Pandemic
}

\author{
Utibe R. Essien, MD, $\mathrm{MPH}^{1,2}{ }^{\oplus}$, Nwamaka D. Eneanya, MD, MPH ${ }^{3,4}$, and Deidra C. Crews, \\ $M D, S C M, 6$
}

\begin{abstract}
'Division of General Internal Medicine, University of Pittsburgh School of Medicine, Pittsburgh, PA, USA; ${ }^{2}$ Center for Health Equity Research and Promotion, VA Pittsburgh Healthcare System, Pittsburgh, PA, USA; ${ }^{3}$ Department of Medicine, Perelman University of Pennsylvania School of Medicine, Philadelphia, PA, USA; ${ }^{4}$ Penn Medicine Palliative and Advanced Illness Research Center, University of Pennsylvania School of Medicine, Philadelphia, PA, USA; ${ }^{5}$ Division of Nephrology, Department of Medicine, Johns Hopkins University School of Medicine, Baltimore, MD, USA; ${ }^{6}$ Johns Hopkins Center for Health Equity, Baltimore, MD, USA.
\end{abstract}

J Gen Intern Med 35(9):2760-2

DOI: $10.1007 / \mathrm{s} 11606-020-05976-\mathrm{y}$

(c) Society ofGeneral InternalMedicine (This is aU.S. government work and not under copyright protection in the U.S.; foreign copyright protection may apply) 2020

$\mathrm{C}$ OVID-19 infection has become widespread, affecting over 4 million individuals worldwide and resulting in nearly 300,000 deaths as of May 15, 2020. ${ }^{1}$ Across the globe, including in the USA, the pandemic has raised concerns for exacerbating social and structural inequities in the health care system. Disparities in chronic cardiopulmonary disease prevalence, socioeconomic status, and access to health care have placed vulnerable populations, including racial and ethnic minorities, at higher risk of COVID-19 infection, hospitalization, and death. Black, Hispanic, and Native Americans have been shown to be infected and dying at disproportionately higher rates than their white counterparts in large urban and rural areas across the USA. ${ }^{2}$ As the number of positive cases rise, so too has the expectation of scarce health care resources including SARS-CoV-2 antigen and antibody testing, antiviral medications, intensive care unit beds, and ventilators, elevating calls by health care leaders and public health officials to develop and implement strategies for rationing of critical resources. ${ }^{3}$ These calls are especially alarming for marginalized populations whom history has shown are persistently excluded from resources, both scarce and abundant, in the USA. As the current pandemic draws into focus the stark variation in access to limited resources, from personal protective equipment to dialysis machines, ${ }^{4}$ we caution that equity must be placed at the center of all rationing strategies, citing examples from organ transplantation and end-of-life care to amplify a call for action to protect vulnerable communities in this health crisis.

Received April 6, 2020

Accepted June 11, 2020

Published online June 30, 2020

\section{LESSONS FROM DISPARITIES IN ORGAN TRANSPLANTATION}

As of today, there are approximately 113,000 individuals awaiting a solid organ transplant in the USA, compared with 39,000 who received a transplant in $2019 .{ }^{5}$ Given the scarcity of this life-saving resource, health systems and national medical societies have established policies to best allocate these organs. ${ }^{6}$ Nevertheless, countless analyses have reported disparities in receipt of organ transplantation by race, ethnicity, income level, insurance, and immigration status. ${ }^{7}$ These disparities may eventually worsen as transplant programs are performing fewer evaluations or have stopped performing transplant surgeries altogether due to limited data related to transplantation and COVID-19. ${ }^{8}$ These well-described disparities, observed in myriad solid organ transplantation types, offer an important example for how rationing in the COVID19 pandemic may unfold in the USA. The reasons attributed to the disparities in organ transplantation are broad ranging, ${ }^{9}$ including clinician referral bias, decreased awareness of transplantation as a therapeutic option, and a higher prevalence of medical comorbidities such as diabetes, hypertension, and obesity in vulnerable groups. Health care clinicians and transplant committee members may also emphasize subjective findings such as limited social support and resource availability as fundamental barriers to transplantation, thus leaving socially vulnerable populations at lower likelihood of getting listed. Policy-based interventions may also miss the mark in addressing disparities. For example, whereas the priority of a new Kidney Allocation System Organ Procurement and Transplantation Network policy was to improve access to transplantation to underserved populations, a recent analysis showed persistent disparities in kidney transplantation, with white patients more likely to move from the "inactive" to the "active" transplant list compared with their minority counterparts. ${ }^{10}$ Without addressing equity early, we could witness a similar story in the COVID-19 pandemic, where determining who has access to testing, medical management, and ultimately vaccination to prevent the disease may differ across dreaded racial and socioeconomic lines. 


\section{LESSONS FROM DISPARITIES IN END-OF-LIFE CARE}

There has been increasing advocacy to optimize end-of-life care for seriously ill patients. Indices to achieve high-quality end-of-life care, including timely referral to palliative care, avoidance of unnecessary and burdensome intensive procedures, and promotion of hospice for terminally ill patients as opposed to ICU admission, have gained favor across all medical subspecialties. This trend is particularly important amid a pandemic that has prompted several health care institutions to develop scoring algorithms to triage whether patients with COVID-19 infections will receive immediate intensive treatment or palliative care and 28 states to develop publicly available crisis standards of care. ${ }^{11}$ In the last days of life, palliative care is beneficial for patients, their loved ones, and even health care workers given the emphasis on treatment of symptoms and emotional support. However, racial and ethnic minorities are less likely to receive palliative care resources and more likely to experience intensive care at the end-of-life. The reasons for this disparity are numerous including lack of palliative care referrals, lower quality communication with clinicians, and limited understanding of the benefits of palliative consultations and hospice care among minority groups. ${ }^{12}$ In addition, racial bias among clinicians can affect whether certain groups of patients receive adequate palliation such as treatment of pain. Battling distrust in the health care system along with fears of medical providers withholding care, racialethnic minorities, and other underserved populations will undoubtedly receive disparate end-of-life care during this crisis. ${ }^{13}$ As hospitals that have reached capacity have already endorsed universal Do-Not-Resuscitate orders for seriously ill patients who are infected with COVID-19, ensuring equity in goals of care and limited life-sustaining treatment discussions is critical. These conversations surrounding end-of-life care will likely be received differentially by vulnerable communities that may not have established trust with the health care system. Furthermore, many may fear that they will be unfairly left to die while not understanding or even being offered endof-life palliative services.

\section{ENSURING EQUITY IN SCARCITY AND AT END-OF-LIFE}

The following months will greatly test the resolve of the health system. Front-line clinicians and health system leaders are making urgent decisions each day that impact thousands of lives. These decisions are uncomfortable and often fall outside of the scope of most US-based medical training. Prioritizing equity in the midst of these decisions will require bold, pragmatic, and creative solutions that address the breadth of care, from the initial presentation to the end of life. We propose several tenets to ensure equity is upheld within clinical approaches. First, granular sociodemographic information, including race and ethnicity, is needed at the hospital, state, and federal level to clearly assess the impact of decision-making on infection rates, mortality, and long-term morbidity. Along these lines, at the policy level, we recommend the creation of state and federal Pandemic Health Equity Accountability Panels. Such panels will help review hospital-level data collected throughout the pandemic including rates of testing, therapeutic decisions, and outcomes, to help ensure that all hospital-wide policies are being applied equally. Second, we propose that institutional crisis triage committees, essential in reducing the stress of rationing decisions by bedside clinicians, ${ }^{6}$ be diverse with regard to race, gender, community, and professional status. In addition to triage decisions, these diverse teams can also support treating clinicians by providing culturally sensitive and patient-centered language for discussing rationing decisions with patients and their families. Third, we encourage health systems to work with community partners to increase awareness around advance care planning and advance directives prior to hospitalization. In addition, given the known national shortage of palliative care specialists, we recommend that all clinicians engage in primary palliative care to address basic goals of care discussions and treatment of symptoms. Finally, given the rapid course of the disease, we recommend early palliative care referral, where possible, for all patients hospitalized with presumed COVID19 infection. We also recommend that out-of-hospital resources, including outpatient clinical volunteers, be redeployed to support critical care staff in having difficult and time-intensive family discussions related to end-of-life care.

History often offers a lens into the future. However, we do not have to look too far back into our nation's history to observe the inequities in health care that are placing the most vulnerable at highest risk during this current pandemic. It is our hope that addressing health equity early will avoid perpetuating disparities and historical trauma and allow us to join together on the other side of this crisis to create a better future of health care for all Americans.

Corresponding Author: Utibe R. Essien, MD, MPH; Division of General Internal Medicine, University of Pittsburgh School of Medicine, Pittsburgh, PA, USA (e-mail: uessien@pitt.edu).

\section{Compliance with Ethical Standards:}

Conflict of Interest: The authors have no conflicts to report.

\section{REFERENCES}

1. World Health Organization. Coronavirus Disease 2019 (COVID-19) Situation Report-115.; 2020. https://www.who.int/docs/defaultsource/coronaviruse/situation-reports/20200514-covid-19-sitrep-115. pdf?sfvrsn=3fce8d3c_6. Accessed 16 May 2020.

2. Williams DR, Cooper LA. COVID-19 and Health Equity-A New Kind of "Herd Immunity". JAMA 2020. doi:https://doi.org/10.1001/jama.2020. 8051

3. Emanuel EJ, Persad G, Upshur R, et al. Fair Allocation of Scarce Medical Resources in the Time of Covid-19. N Engl J Med. 2020:NEJMsb2005114. doi:https://doi.org/10.1056/NEJMsb2005114

4. New York Times. An Overlooked, Possibly Fatal Coronavirus Crisis: A Dire Need for Kidney Dialysis. https://www.nytimes.com/2020/04/18/ health/kidney-dialysis-coronavirus.html. Published 2020. Accessed 15 May 2020. 
5. United Network for Organ Sharing. Transplant trends. https://unos.org/ data/transplant-trends/. Published 2020. Accessed 31 March 2020.

6. White DB, Lo B. A Framework for Rationing Ventilators and Critical Care Beds During the COVID-19 Pandemic. JAMA 2020. doi:https://doi.org/ 10.1001/jama.2020.5046

7. Purnell TS, Luo X, Cooper LA, et al. Association of race and ethnicity with live donor kidney transplantation in the United States From 1995 to 2014. JAMA J Am Med Assoc 2018. doi:https://doi.org/10.1001/jama. 2017.19152

8. Boyarsky BJ, Chiang TPY, Werbel WA, et al. Early Impact of COVID-19 on Transplant Center Practices and Policies in the United States. Am J Transplant 2020. doi:https://doi.org/10.1111/ajt.15915

9. Morris AA, Kransdorf EP, Coleman BL, Colvin M. Racial and ethnic disparities in outcomes after heart transplantation: A systematic review of contributing factors and future directions to close the outcomes gap. J Heart Lung Transplant 2016. doi:https://doi.org/10.1016/j.healun. 2016.01.1231

10. Kulkarni S, Ladin K, Haakinson D, Greene E, Li L, Deng Y. Association of Racial Disparities with Access to Kidney Transplant after the
Implementation of the New Kidney Allocation System. JAMA Surg 2019. doi:https://doi.org/10.1001/jamasurg.2019.0512

11. Cleveland Manchanda E, Couillard $\mathbf{C}$, Sivashanker $\mathbf{K}$. Inequity in Crisis Standards of Care. N Engl J Med. 2020:NEJMp2011359. doi:https://doi.org/10.1056/NEJMp2011359

12. Eneanya ND, Wenger $\mathbf{J B}$, Waite $\mathbf{K}$, et al. Racial disparities in end-of-life communication and preferences among chronic kidney disease patients. Am J Nephrol 2016. doi:https://doi.org/10.1159/000447097

13. Conley C Racial health disparities already existed in America- the coronavirus just exacerbated them. CNBC. https://www.cnbc.com/ 2020/05/14/how-covid-19-exacerbated-americas-racial-health-disparities.html. Published 2020. Accessed 15 May 2020

Publisher's Note: Springer Nature remains neutral with regard to jurisdictional claims in published maps and institutional affiliations. 